\title{
PERCEPCIONES Y EXPERIENCIAS DEL INICIO SEXUAL DESDE UNA PERSPECTIVA DE GÉNERO, EN ADOLESCENTES CONSULTANTES EN UN CENTRO UNIVERSITARIO DE SALUD SEXUAL Y REPRODUCTIVA
}

\author{
Electra González A. ${ }^{1 a}$, Adela Montero V. ${ }^{1}$, Vania Martínez N. ${ }^{1}$, Pamela Mena G. ${ }^{\text {, }}$ \\ Marioli Varas L. ${ }^{b}$ \\ ${ }^{1}$ Centro de Medicina Reproductiva y Desarrollo Integral de la Adolescencia (CEMERA), Facultad de Medicina, \\ Universidad de Chile.
}

a Asistente Social, ${ }^{b}$ Alumnas, Escuela de Medicina, Universidad de Chile.

\section{RESUMEN}

Antecedentes: El contexto en que se inicia la actividad sexual en adolescentes tiene importantes significados para las personas e implicancias en salud pública. Objetivo: Conocer con un enfoque exploratorio, los factores que influyen en el inicio de la actividad sexual en adolescentes y explorar el contexto en el cual ocurre. Método: Muestra de adolescentes, hombres y mujeres, sexualmente activos entre 14 y 19 años, reclutados en el CEMERA, durante 2007. Se realizaron 117 entrevistas semiestructuradas. Se utilizó el análisis temático de los datos. Los significados e interpretaciones fueron constantemente verificados a través de sucesivos análisis. Resultados: El grado de control personal que tuvieron sobre los factores que condujeron a su primera experiencia sexual, determinó como ellos/as se sintieron con esta experiencia. Aquellos/as que dijeron que había sido una buena experiencia, demoraron el inicio sexual hasta que se sintieron cómodos/as con el momento y con quien debutaron sexualmente. Sin embargo, sentimientos de culpa marcaron el evento, porque ellos/as habían transgredido las normas sociales. En contraste, la coerción de sus parejas y los efectos del alcohol, fueron razones que llevaron a una experiencia sexual prematura y no deseada. Conclusiones: El rol de la interacción con sus pares, el abuso de alcohol y la presión de la pareja, en el caso de las niñas, influyen en la actividad sexual prematura y no deseada. Las intervenciones educacionales debieran considerar estos aspectos para que tengan relevancia personal y el impacto pueda ser mayor en la postergación del inicio de la actividad sexual o que esta sea protegida.

\section{PALABRAS CLAVE: Adolescencia, inicio sexual, comportamiento sexual, género}

\section{SUMMARY}

Background: It is important to understand that the timing and context in which sexual intercourse is initiated, has significant personal and public health implications. Objective: To explore the factors that influence the initiation of first intercourse among adolescent females and males and the context in which this event occurs. Methods: A sample of sexually active teenagers both female and male, aged between 14 and 19 years, were recruited from a University Centre for Sexual and Reproductive Health Care (CEMERA), during 2007. One hundred and seventeen semi structural interviews were conducted. Thematic analysis was used to analyze the data. Meanings and interpretations that emerged were constantly verified through subsequent inquiry. 
Results: The degree of personal control over the factors that led to their first experience of intercourse determined how adolescents reflected upon the experience. Those who were ready and felt well were more likely to have delayed intercourse until they were comfortable with when and with whom the sexual debut occurred. However, feelings of blame because the social norms were transgressed marked the event. In contrast, coercion from sexual partners and being under effect of alcohol were reasons for premature and unwanted first experience of sexual intercourse. Conclusions: The role of peer interaction and abuse of alcohol, and the pressure from sexual partner to influence in premature and unwanted sexual activity, may to generate educational interventions of greater personal relevance and impact to prevent risky sexual behaviors.

\section{KEY WORDS: Adolescence, sexual debut, sexual behavior, gender}

\section{INTRODUCCIÓN}

En Chile, como en muchos países, los y las adolescentes inician actividad sexual a edades mas tempranas que en generaciones anteriores (1). La evidencia científica muestra que el inicio de la actividad sexual a más temprana edad puede tener consecuencias psicológicas, sociales y económicas negativas (1-3). El inicio sexual temprano esta asociado también a un mayor número de parejas sexuales a lo largo de la vida. Estas tendencias tienen una influencia enorme en la ocurrencia de comportamientos sexuales de riesgo y en la epidemiología del embarazo adolescente, como también en las infecciones de transmisión sexual y VIH-SIDA (1-8).

Reducir el embarazo adolescente y las infecciones de transmisión sexual, incluida el SIDA, son metas de políticas públicas en nuestro país, pero hasta ahora las estrategias han sido insuficientes y poco efectivas.

El tiempo y el contexto en el cual ocurre el debut sexual tienen importantes implicancias tanto en el significado personal de los individuos como en salud pública. Diversos estudios muestran que la maduración física más temprana, las percepciones de actitudes positivas hacia la actividad sexual, y una percepción de alta prevalencia de actividad sexual entre los pares, el inicio temprano de las relaciones románticas, el uso temprano y problemático de alcohol y otras drogas, son factores determinantes del inicio de la actividad sexual temprana (9-17). La mayoría de estos resultados han sido generados a través de investigaciones con metodología cuantitativa (18-30), sin embargo, este enfoque no logra profundizar en el contexto psicosocial, en el cual se da el debut, ni en los significados que los y las adolescentes le asignan a esta experiencia. Es necesario para ello emplear una metodología cualitativa que logre capturar con mayor profundidad este complejo contexto.

El objetivo de este estudio es conocer cuales son los procesos sociales en el cual se produce la primera experiencia sexual, y los significados que los y las adolescentes adscriben a este evento desde la experiencia misma de los y las participantes.

\section{MATERIAL Y MÉTODO}

El diseño corresponde a un estudio cualitativo. La muestra del estudio estuvo constituida por adolescentes de ambos sexos, entre 14 y 19 años, sexualmente activos, que consultaron en CEMERA en el año 2007. No se consideró como debut sexual el abuso sexual infantil. Los y las adolescentes firmaron un consentimiento informado aprobado por el Comité de Ética de la Facultad de Medicina de la Universidad de Chile, previo a la aplicación de la entrevista semi-estructurada diseñada para el estudio. Se exploró el contexto, las creencias y experiencias de los y las participantes en relación al inicio sexual, relaciones románticas, actividad sexual, y como se sintieron con esta experiencia. Las preguntas fueron: ¿Cuáles fueron las razones o motivos que te llevaron a tener relaciones sexuales la primera vez?, ¿Qué significó para ti la primera vez?, ¿Cómo te sentiste después de esta experiencia? La actividad sexual fue consignada como haber tenido actividad sexual coital vaginal para asegurar consistencia en la interpretación de lo que llamaremos primera relación sexual o debut sexual.

Análisis de la información: Se realizó un análisis temático y los temas que emergieron se discutieron, se compararon y modificaron hasta llegar a categorías conceptuales. Se realizó una interpretación de los datos manteniendo lo más posible la objetividad de los eventos y verdaderos significados de la información entregada por las y los participantes del estudio.

\section{RESULTADOS}

Un total de 117 entrevistas fueron realizadas (72 mujeres y 45 hombres). Los antecedentes demográficos se presentan en Tabla I. 
Tabla I

DISTRIBUCIÓN PORCENTUAL Y PROMEDIOS DE LAS CARACTERÍSTICAS DEMOGRÁFICAS DE ADOLESCENTES PARTICIPANTES POR SEXO $(n=117)$

\begin{tabular}{|c|c|c|c|c|}
\hline \multirow[b]{2}{*}{ Variables } & \multicolumn{2}{|c|}{ Hombres $(n=45)$} & \multicolumn{2}{|c|}{ Mujeres $\quad(n=72)$} \\
\hline & Frecuencia & $\%$ & Frecuencia & $\%$ \\
\hline \multicolumn{5}{|l|}{ Edad a la entrevista (años) } \\
\hline $14-15$ & 6 & 13,3 & 27 & 37,5 \\
\hline $16-17$ & 16 & 35,6 & 45 & 62,5 \\
\hline $18-19$ & 23 & 51,1 & - & - \\
\hline \multicolumn{5}{|l|}{ Escolaridad (años cursados) } \\
\hline$\leq 8$ & 1 & 2,2 & 5 & 6,9 \\
\hline 9 a 10 & 12 & 26,7 & 27 & 37,5 \\
\hline 11 a 12 & 25 & 55,6 & 40 & 55,6 \\
\hline$>12$ & 7 & 15,5 & - & - \\
\hline \multicolumn{5}{|l|}{ Vive con: } \\
\hline Ambos padres & 25 & 55,6 & 32 & 44,4 \\
\hline Madre y padrastro & 2 & 4,4 & 13 & 18,1 \\
\hline Madre sola & 14 & 31,1 & 19 & 26,4 \\
\hline Padre sólo & 3 & 6,7 & 3 & 4,2 \\
\hline Otro & 1 & 2,2 & 5 & 7,0 \\
\hline \multicolumn{5}{|l|}{ Debut sexual con: } \\
\hline Novio (a) & 32 & 71,1 & 63 & 87,5 \\
\hline Amigo/a & 4 & 8,9 & 1 & 1,4 \\
\hline Pareja casual & 5 & 11,1 & 7 & 9,7 \\
\hline Ocasional & 4 & 8,9 & 1 & 1,4 \\
\hline \multicolumn{5}{|l|}{ Edad al debut sexual: } \\
\hline 13 años & 8 & 17,8 & 10 & 13,9 \\
\hline 14 años & 7 & 15,6 & 12 & 16,7 \\
\hline 15 a 16 años & 16 & 35,6 & 41 & 56,9 \\
\hline$\geq 17$ & 14 & 29,0 & 9 & 12,5 \\
\hline \multicolumn{5}{|l|}{$\begin{array}{l}\text { Edad a la primera relación } \\
\text { romántica (años) }\end{array}$} \\
\hline$<13$ & 8 & 17,7 & 18 & 25,0 \\
\hline 13 a 14 & 22 & 48,9 & 41 & 57,0 \\
\hline 15 a 16 & 12 & 26,7 & 13 & 18,1 \\
\hline$\geq 17$ & 3 & 6,7 & - & - \\
\hline $\begin{array}{l}\text { Edad pareja debut sexual } \\
\text { (promedio en años) }\end{array}$ & 15,5 & & 17,5 & \\
\hline $\begin{array}{l}N^{\circ} \text { parejas sexuales } \\
\text { (promedio) }\end{array}$ & & & 10 & \\
\hline Tiempo relación pareja & & & & \\
\hline $\begin{array}{l}\text { al debut sexual (promedio en } \\
\text { meses) }\end{array}$ & 3,8 & & 6,5 & \\
\hline
\end{tabular}


Nuestro análisis desarrolló varias áreas temáticas que ofrecieron insight en los factores que influyeron en el inicio de la actividad sexual de los y las adolescentes del estudio, y el contexto en el cual este evento ocurrió. Para la mayoría de los y las adolescentes las primeras experiencias sexuales coincidieron con el cambio desde el colegio bási$\mathrm{co}$ al liceo. Sus primeros encuentros con el sexo opuesto son con otros escolares de colegios cercanos y son descritos como "inocentes", "entretenidos", a menudo producto de la curiosidad, tomarse de la mano, besarse y tener su primera relación romántica. Mayores oportunidades y deseos de experimentar en forma progresiva en los comportamientos sexuales se fue dando especialmente en las "discoteques" diurnas, algunas instaladas muy cercas de los establecimientos escolares donde asisten.

Recordando su primera experiencia sexual y el contexto en que se desarrolló emergieron las siguientes categorías:

1) Adolescentes que lo hicieron "por amor", "por estar enamorado", una adolescente recalcó que "estaba súper enamorada y quería que él, fuera mi primer hombre" como el principal motivo por el cual iniciaron actividad sexual.

2) Adolescentes que lo hicieron por "curiosidad" y "por saber que se sentía". "Tú sólo quieres experimentar cómo es". Incluso para aquellos(as) que no estaban en una relación romántica en ese momento, su curiosidad los(as) llevó a tener actividad sexual con un amigo(a) porque sintieron que era más seguro que con un desconocido(a). "Mi primera vez fue con una amiga a quien conocía desde hacia años". Las percepciones de que la actividad sexual es algo grandioso es algo que escuchan de sus pares, "la Jenny nos contó a todo el curso lo bien que lo había pasado". En este sentido las conversaciones con sus amigas emergieron como una fuente importante de querer saber sobre eso. La curiosidad hacia la actividad sexual coincidió con expectativas altas construidas en relación a este evento. Una adolescente dijo: "me hice hartas ilusiones la primera vez...", sostener expectativas idealizadas del encuentro físico en si y nociones no realistas del significado asignado al sexo, deja algunos sentimientos molestos con la primera experiencia: "todo lo que yo sentí fue el dolor, la incomodidad de perder mi virginidad".

3) Adolescentes que lo hicieron porque experimentaron un deseo interno por la aprobación de los pares. Una adolescente remarcó: "es mucha la presión de los pares, la gente conversa acerca de eso en la escuela y tú estas afuera de las conversaciones a menos que lo hagas". Es necesario para mantener su status en el grupo de pares, conversar acerca del tema a través de su propia experiencia. Conversar de sexualidad entre los amigos y compañeros, crea la percepción de que la mayoría de ellos ya ha tenido actividad sexual, que ellos "se están perdiendo algo", o "sentirse nerd por ser virgen todavía". "Me sentía como imbécil todos lo habían hecho menos yo" remarcó un adolescente quien tuvo su primera experiencia sexual a los 17 años.

4) Adolescentes que lo hicieron estando bajo efectos del alcohol. Lo que consideraron como una razón legítima para el control limitado que tuvieron en su debut sexual, "pasó como chacota fue la volada del copete" se justificó un adolescente, o pueden sentirse más débil, "una está más débil con tragos y es más difícil decir que no". El estar bajo efectos del alcohol reduce las inhibiciones y la racionalidad y hace que la progresión hacia la actividad sexual sea más fácil. "No debería haberlo hecho, estaba en una fiesta, tomé mucho, no me di cuenta como pasó".

5) Adolescentes que lo hicieron sin tener una motivación especial: "estábamos solos, nos dejamos llevar", o "fue la excitación del momento".

6) Adolescentes que lo hicieron cuando se sintieron preparados. Las adolescentes recordaron su primera vez en una manera más positiva, estaban contentas en cómo ocurrió y con quien ocurrió. Las adolescentes que postergaron más tiempo la relación sexual en una relación de pareja reportaron sentirse preparadas, "Yo esperé con mi pololo harto tiempo hasta que yo me sentí segura y preparada, yo tenía confianza en él "sentimientos de estar preparada" y sentirse "cómoda" implicó que ellas tuvieran el control en el debut sexual. Además, lo vieron como algo natural en su relación "fue el momento apropiado para mi y con la persona que yo quería". Una comunicación abierta y negociación mutua aseguró la decisión de tener relaciones sexuales, fue una responsabilidad compartida. Este tema sugiere la importancia de "saber" y "confiar" en su pareja antes de tener relaciones con él.

7) Adolescentes que lo hicieron bajo cierta coerción. En contraste con aquellas que se sintieron listas para el inicio de la actividad sexual, otras adolescentes recordaron que lo hicieron en cierta forma coercionadas: "yo no quería tener relaciones pero cedí, después de eso terminamos". Aquí vemos las dificultades que tienen algunas niñas para negociar efectivamente el comportamiento sexual con sus parejas. Sentirse coercionadas fue común en ellas, particularmente en aquellas que se iniciaron a edades más jóvenes: "tú lo haces porque él quiere", "sólo lo hice para que él se sintiera feliz" o "él me dijo que le diera la prueba de amor", implica 
la naturaleza sumisa de las adolescentes en sus relaciones y su experiencia de actividad sexual no deseada.

La necesidad de mantener la relación e impedir conflictos con su pareja fue importante para otras participantes del estudio. Esto fue evidente en adolescentes de menor edad, con parejas mayores o que no vivían con sus padres. El sexo es visto como algo que mantendría a los pololos interesadas en ellas especialmente en carencias afectivas familiares: "él me entregó cariño y fue muy importante para mi”. En estos casos el miedo a que su pareja la dejara impidió a estas adolescentes posponer la actividad sexual. También los varones pueden sentirse presionados a la actividad sexual "ella me presionó y para no quedar mal..." expresó un adolescente que inicio su actividad sexual a los 14 años con una amiga de 15.

¿Qué significó para ti tu primera experiencia sexual?

Fue "algo muy lindo", "muy importante", "enriquecedor", "algo especial" en sus vidas especialmente aquellos(as) que sintieron que "era el momento correcto y con la persona correcta". Un adolescente expresó que fue "bacán, una sensación que nunca había sentido". Mientras que aquellos y aquellas que fueron motivados(as) por el deseo, o sólo por experimentar significó un "paso más en la vida", o "nada especial", o una sensación de error como expresó una adolescente "una pierde harto".

¿Cómo te sentiste después de esta experiencia?

A) Adolescentes de ambos sexos expresaron sentimientos agradables de esta experiencia en el contexto de una relación romántica: "me sentí más en contacto con él", "me sentí querida, amada", "sentí que la quería más", "lo más bonito que me ha pasado en la vida".

B) Adolescentes que expresaron sentirse "rara como que no era yo" y varones que se sintieron "raro porque no sabia como actuar frente a ella".

C) Adolescentes varones que expresaron no haber sentido "nada" o "no fue como yo pensaba"

D) Adolescentes que expresaron vergüenza: "me daba vergüenza mirarlo".

E) Adolescentes que expresaron preocupación, temor y culpa a pesar que el debut sexual se dio en un contexto adecuado y en una relación romántica: "el temor al embarazo", "por el tema de la iglesia que no permite las relaciones antes del matrimonio", "porque me cambiaría el cuerpo y se darían cuenta mis padres", "se me vino mi papá a mi cabeza", "asustada, no sabía como contarle a mis padres" y finalmente "asustada, porque sangré bastante y no sabía que hacer".
F) Adolescentes que sintieron arrepentimiento: "arrepentida por mi mamá", "hubiera preferido esperar, pienso que fue muy temprano", "porque fue puro dolor", "me sentí tonta, no me gustó para nada".

G) Adolescentes varones que dijeron sentirse "mal" porque tenían dudas acerca de su desempeño, como lo expresó un adolescente de 16 años: "tenía dudas si lo había hecho bien o mal".

\section{DISCUSIÓN}

Nuestro estudio localiza el debut sexual en un marco teórico biopsicosocial, esto significa que el tiempo de la primera relación sexual se correlaciona con una compleja red de características personales e influencias sociales que moldean la decisión de los y las adolescentes por iniciar o retrasar el inicio sexual.

El control sobre los factores que determinan la primera relación sexual tuvo una particular importancia en como los y las adolescentes vivieron su primera experiencia sexual y el significado que adscribieron al encuentro. Estos hallazgos apoyan que el sentido del control personal como una importante influencia en el tiempo y la naturaleza del debut sexual. Nuestros datos revelaron patrones de discurso que reflejaron elecciones activas y pérdidas de control por sobre las razones para el inicio de la actividad sexual.

El discurso de las adolescentes que pospusieron la actividad sexual hasta el contexto de "era el tiempo correcto y con la persona correcta”, mostró patrones de decisión personal racionales que las hicieron reflexionar en sus percepciones de experiencias a la edad apropiada y de autodesarrollo. Descripciones de "esperar" y sentimiento de "correcto" refleja una elección conciente y deseo de comprometerse en la primera relación, y son sinónimos de la noción de estar "listas" para la transición desde la abstinencia en un proceso del desarrollo natural. También reveló que características individuales como la edad, actitud y creencias en conjunto con "estando en una relación amorosa estable”, son claves para la decisión sexual en estas participantes.

Otro discurso reveló experiencias más bien decepcionantes y culposas en aquellas adolescentes que iniciaron más tempranamente la actividad sexual y por razones externas a ellas. La influencia del alcohol y presión de la pareja emergieron de las experiencias de las adolescentes como un discurso en el cual la actividad sexual fue concebida como un evento pasivo y no relacionada con una intencionalidad personal. Comentarios "sólo pasó", 
"sólo sucedió", "se dio el momento", indicaron un bajo control del momento en que ocurrió el debut sexual y entró en conflicto con las expectativas de esta transición como un evento "especial", especialmente en las mujeres.

Es importante señalar el rol que cumplen los pares en el inicio sexual. Estudios previos han mostrado una fuerte asociación entre las normas sociales y el comportamiento adolescente. Específicamente, el comportamiento sexual de los amigos, la percepción de los comportamientos y actitudes de los amigos, $y$ el nivel de compromiso con ellos han sido identificados como factores protectores y de riesgo claves para la actividad sexual temprana.

Este estudio agrega mayor información de los procesos sociales, subrayando las situaciones complejas y la construcción de percepciones y como los y las adolescentes responden a normas sociales en un contexto del desarrollo. A mayor control sobre las circunstancias que rodean el debut sexual menor probabilidad que localicen sus experiencias en el dominio de las normas sociales.

La influencia de las parejas apareció más fuerte entre las iniciadoras más tempranas, sugiriendo la importancia de la interacción entre el contexto social y el desarrollo adolescente. En los varones, conceptos como sentido de pertenencia y aceptación de los pares, se reflejó a través del deseo de ser "como uno de ellos", lo que los lleva a formar parte de las expectativas y comportamientos del grupo. En este sentido, la dinámica natural de las interacciones con los pares durante la adolescencia y el aumento de las conversaciones acerca de la actividad sexual, moldeó sus percepciones de las actitudes y comportamientos normativos y su propia curiosidad hacia la actividad sexual.

En las adolescentes iniciar actividad sexual antes de sentirse preparadas en el manejo de las consecuencias emocionales y sociales, tiene potenciales implicaciones tales como embarazo no deseado e infecciones de transmisión sexual, y en los varones en sentirse inseguros en su desempeño sexual.

\section{CONCLUSIONES}

El rol de la interacción de los adolescentes con sus pares, el abuso de alcohol y la presión de la pareja, en el caso de las niñas, influyen en la actividad sexual prematura y no deseada, por lo que las intervenciones educacionales debieran considerar estos aspectos para que tengan relevancia personal, y el impacto pueda ser mayor en la postergación del inicio de la actividad sexual, o que esta sea protegida.

\section{BIBLIOGRAFÍA}

1. González E, Molina T, Montero A, Martínez V. Comportamientos sexuales y diferencias de género en adolescentes usuarios de un sistema público de salud universitario. Rev Med Chile 2007;135:1261-9.

2. Hogan D, Nan MA. The transition to adulthood. Ann Rev Sociol 1986;12:109-30.

3. Reiss L, Premarital sex as deviant behavior: An application of current approaches to deviance. Am Sociol Rev 1970;35:78-87.

4. O"Donnell L,O"Donnell C R, Stueve A. Early sexual initiation and subsequent sex-related risks among urban minory youth: the reach for health study. Fam Plann Perspect 2001; 33(6):268-275.

5. Hayes Ch (Eds). Risking the future: Adolescent sexuality, pregnancy and childbearing 1987;1,Washington, DC: National Academy Press.

6. Hofferth S. "Factors affecting initiation of sexual intercourse". In: Risking the future: Adolescent sexuality, pregnancy and childbearing. Washington, DC: National Academy Press 1987;1:7-35.

7. Molina R, Sandoval J. González E. (Ed). Salud sexual y reproductiva en la adolescencia. Editorial Mediterráneo, 2003.

8. Forste R, Heator TB. Initiation of sexual activity among female adolescents. Youth Soc 1988;19:250-68.

9. Miller B, Olson TD. Sexual attitudes and behaviour of high school students in relation to background and contextual factors. J Sex Research 1988;24:194-200.

10. Gowen LK, Feldman SS, Diaz R, Yisrael DS. A comparison of the sexual behavior and attitudes of adolescent girls with older vs. similar-aged boyfriends. J Youth Adol 2004; 33:167-75.

11. Vanoss Marín B, Coyle KK, Gómez CA, Carvajal SC, Kirby DB. Older boyfriends and girlfriends increase risk of sexual initiation in young adolescents. J Adolesc Health 2000;27(6):409-18.

12. Miller B, McCoy JK, Olson TD. Dating age and stage as correlates of adolescent attitudes and behavior. $J$ Adol Res 1986;1:361-71.

13. Thornton A. The courtship process and adolescent sexuality. J Family Issues 1990;11:239-73.

14. Jorgensen S, King SL, Torrey BA. Dyadic and social network influences on adolescent exposure to pregnancy risk. J Marriage Fam 1980;42:141-55.

15. Billy GJ, Rodgers LJ, Udry JR. Adolescent sexual behavior and friendship choice. Social Forces 1984;62(3):653-78.

16. McBride MV. Black adolescent females: a comparison of early versus late coital initiators. Family Relations 1994;43:342-8.

17. Reiss L, Miller B. Heterosexual permissiveness: a theoretical analysis. In: Burr WR, Hill R, Nye FI, Reiss IL (Eds). Contemporary theories about the family. New York; Free Press,1979. pp 57-100.

18. Lammers C, Ireland M, Resnick M, Blum R. Influences on adolescents' decision to postpone onset of sexual intercourse: a survival analysis of virginity among youths aged 13 to 18 years. J Adol Health 2000;26:428.

19. Hogan PD, Kitawa ME. The impact of social status, 
family structure, and neighborhood on the fertility of black adolescents. AM J Sociol 1985;90:825-55.

20. Mueller KE, Powers WG. Parent-child sexual discussion: perceived communicator style and subsequent behavior. Adolescence 2005;25:469-82.

21. Furstenberg F, Moore KA, Peterson JL. Sex education and sexual experience among adolescents. Am J Public Health 1985;75:1331-2.

22. Fernández L, Bustos L, González L, Palma D, Villagrán J, Muñoz S. Creencias, actitudes y conocimientos en educación sexual. Rev Med Chile 2000;128:574-83.

23. Newcomer FS, Udry JR. Parent-child communication and adolescent sexual behavior. Fam Plann Perspect 1985;17:169-74.

24. Montenegro H. Educación sexual de niños y adolescentes. Rev Med Chile 2000;128:571-3.

25. DeLamater J, MacCorquodale P. Premarital sexuality.
Madison. University of Wisconsin Press.1979.

26. Chilman CS. Coital behavior of adolescents in the United States: A summary of research and implication for further studies. Paper presented at the Annual Meeting of the American Psychological Association, 1983.

27. Zelnick M, Kartner J F. Sexual activity, contraceptive use and pregnancy among metropolitan area teenagers: 1971-1979. Fam Plann Perspect 1980;12:230-7.

28. Forste R, Heaton TB. Initiation of sexual activity among female adolescents. Youth Soc 1988;19:25068.

29. Miller B,Sneesby KR. Educational correlates of adolescent's sexual attitudes and behavior. J Youth Adol 1988;17:521-30.

30. Miller SK, Forehand R, Kotchick AB. Adolescent sexual behavior in two ethnic minors samples: the role of family variables. J Marriage Fam 1999;61:85-98. 